Vol. 13, $n^{\circ} 1 \mid 2009$

Varia

\title{
La noyade judiciaire dans la République de Genève (1558-1619)
}

The Herman Diedericks Prize Essay for 2008 / Lauréat du prix Herman Diederiks 2008

\section{Sonia Vernhes Rappaz}

\section{(2) OpenEdition} Journals

Édition électronique

URL : https://journals.openedition.org/chs/686

DOI : $10.4000 /$ chs.686

ISSN : 1663-4837

Éditeur

Librairie Droz

Édition imprimée

Date de publication : 1 mars 2009

Pagination : 5-23

ISBN : 978-2-600-01295-9

ISSN : 1422-0857

Référence électronique

Sonia Vernhes Rappaz, "La noyade judiciaire dans la République de Genève (1558-1619) », Crime, Histoire \& Sociétés / Crime, History \& Societies [En ligne], Vol. 13, $n^{\circ} 1$ | 2009, mis en ligne le 01 mars 2012, consulté le 22 mars 2022. URL : http://journals.openedition.org/chs/686 ; DOI : https://doi.org/ $10.4000 /$ chs. 686 


\title{
The Herman Diedericks Prize Essay for 2008 Lauréat du prix Herman Diederiks 2008
}

\author{
La noyade judiciaire \\ dans la République de Genève (1558-1619)
}

\author{
Sonia Vernhes Rappaz ${ }^{1}$
}

\begin{abstract}
Sous l'Ancien Régime la mise à mort par noyade n'est mentionnée qu'occasionnellement dans les archives judiciaires européennes. Pourtant, de 1558 à 1620, la justice criminelle genevoise recourt régulièrement à cette pratique exécutive pour sanctionner les crimes «contre nature». Au $X V I^{e}$ siècle, Genève, cité indépendante depuis 1525 et acquise à la Réforme depuis 1536, devient un refuge pour les réformés fuyant les persécutions religieuses françaises ou italiennes. Le durcissement du contrôle social et moral entraîne une sévérité accrue de la part des autorités civiles et favorise l'augmentation du nombre des poursuites pénales contre toute personne coupable de comportement déviant. À la demande des magistrats, des juristes rédigent des "Avis de droit» pour qualifier le crime ou motiver la peine; ces théoriciens du droit se réfèrent aussi bien au jus romanum qu'au Décalogue pour étayer leur argumentation. Exclusion, publicité, exemplarité ou rétributivité: quel que soit le mode d'exécution, la peine capitale condense les caractères spécifiques aux pénalités d'Ancien Régime. Le recours systématique à une nouvelle technique de mise à mort apporte-t-il une dimension supplémentaire à la grammaire visuelle de la peine? Symbolisme purificateur ancestral, influences juridiques étrangères, exécution miroir du crime ou nouveau vocabulaire pénal à l'attention des crimes indicibles, la noyade judiciaire sanctionne un contentieux criminel de délits «contre nature». La réflexion pénale dont elle résulte prend place dans un temps de transition entre jurisprudences médiévale et moderne.
\end{abstract}

Execution by drowning is only occasionally mentioned in the judicial archives of Old Regime Europe. Between 1558 and 1620, however, the criminal justice system of Geneva turned regularly to this practice to punish crimes 'against nature'. During the XVI $I^{\text {h }}$ century Geneva, an independent city since 1536 and Protestant since 1536, became the refuge for Protestants fleeing from religious persecution in France and Italy. An increasingly severe policy of social and moral control on the part of the civil authorities led to an increase in penal proceedings against deviant behaviour. At the request of the magistrates, jurists prepared legal opinions to define crimes and to prescribe punishments; these legal theorists referred as much to the jus romanum as to the Decalogue for their arguments. Whether the intention was to make an example, to provide retribution, exclusion or publicity, capital punishment

Cet article synthétise un mémoire de licence dirigé par le Professeur Michel Porret et soutenu en juin 2007 à la Faculté des Lettres de l'Université de Genève. Doctorante à l'Université de Genève, Sonia Vernhes Rappaz prépare une thèse en histoire moderne intitulée: "Qualifier le crime, motiver la peine, réguler l'inquisitoire: Les "Avis de droit" dans la République de Genève (XVI" siècle)». 
encapsulated the specific characteristics of punishment during the Old Regime. Did the systematic recourse to a new means of execution bring an additional dimension to the visual grammar of punishment? Whether seen as an ancient symbol of purification, the influence of foreign judicial practice, punishment to mirror the offence or a new penal vocabulary, judicial drowning was seen as punishing unspeakable crimes 'against nature.' The penal thought within which it originated is situated during the time of transition from medieval to modern jurisprudence.

\section{INTRODUCTION}

ans toute l'Europe du XVI ${ }^{e}$ siècle, l'«éclat des supplices » et la diversité des peines appliquées témoignent de la montée en puissance du monopole que l'État exerce sur le droit de punir ${ }^{2}$. La roue, le bûcher, le gibet ou le glaive sont les instruments privilégiés d'une justice expiatoire motivée par l'arbitraire des juges. Parmi le catalogue des peines afflictives, la noyade judiciaire n'est pas le mode d'exécution le plus fréquemment prononcé, bien que son usage soit attesté par différents auteurs. Jeté à l'eau dans un sac, noyé la corde au cou, submergé puis enterré, dans son Dictionnaire de la Pénalité, M.B. Saint Edme recense les différents types de noyades appliqués de l'Antiquité au XVI ${ }^{\mathrm{e}}$ siècle ${ }^{3}$. En Bavière, une chanson populaire rapporte qu'une jeune fille condamnée pour infanticide préfère être noyée plutôt que de devoir épouser le bourreau en charge de l'exécution, alors qu'à Amsterdam des exécutions par noyade sont signalées jusqu'en $1730^{4}$. Il subsiste peu de représentations iconographiques d'un tel supplice, à l'exception de miniatures de la fin du $\mathrm{XV}^{\mathrm{e}}$ siècle qui montrent des exécutions par noyade dans les villes de Lucerne ou de Constance ${ }^{5}$. Dans les mêmes régions, de 1520 à 1530 , au plus fort de la répression à l'encontre des Anabaptistes ${ }^{6}$, nombre d'accusés sont exécutés par noyade après la publication de l'édit de Zurich: celui-ci stipule qu'aucun individu - homme, femme ou jeune fille - ne peut se baptiser sous peine d'être arrêté, jugé et condamné à être noyé ${ }^{7}$. Ainsi, bien que mentionnés au gré des procédures criminelles, des textes littéraires ou des articles du Code criminel de la Caroline, les cas de noyade judiciaire apparaissent rares et disséminés et jusqu'à ce jour, les motivations et les circonstances d'application d'une telle peine n'ont pas été l'objet d'une recherche systématique.

De 1558 à 1620, à Genève, république indépendante et acquise à la Réforme depuis peu, la noyade judiciaire est régulièrement appliquée et devient partie intégrante du système répressif local. Il s'agit d'un mode d'exécution qui sanctionne spécifiquement des crimes «contre nature» ou des transgressions sexuelles «aggravées », délits au sujet desquels les juges réclament une très grande discrétion. Infanticides, incestes, viols d'enfants, adultères ou sodomies, le contentieux criminel de

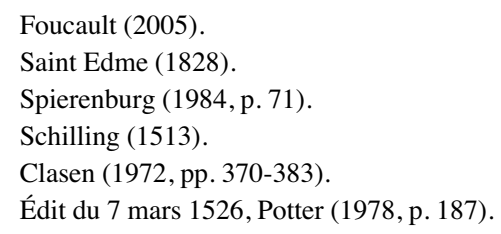




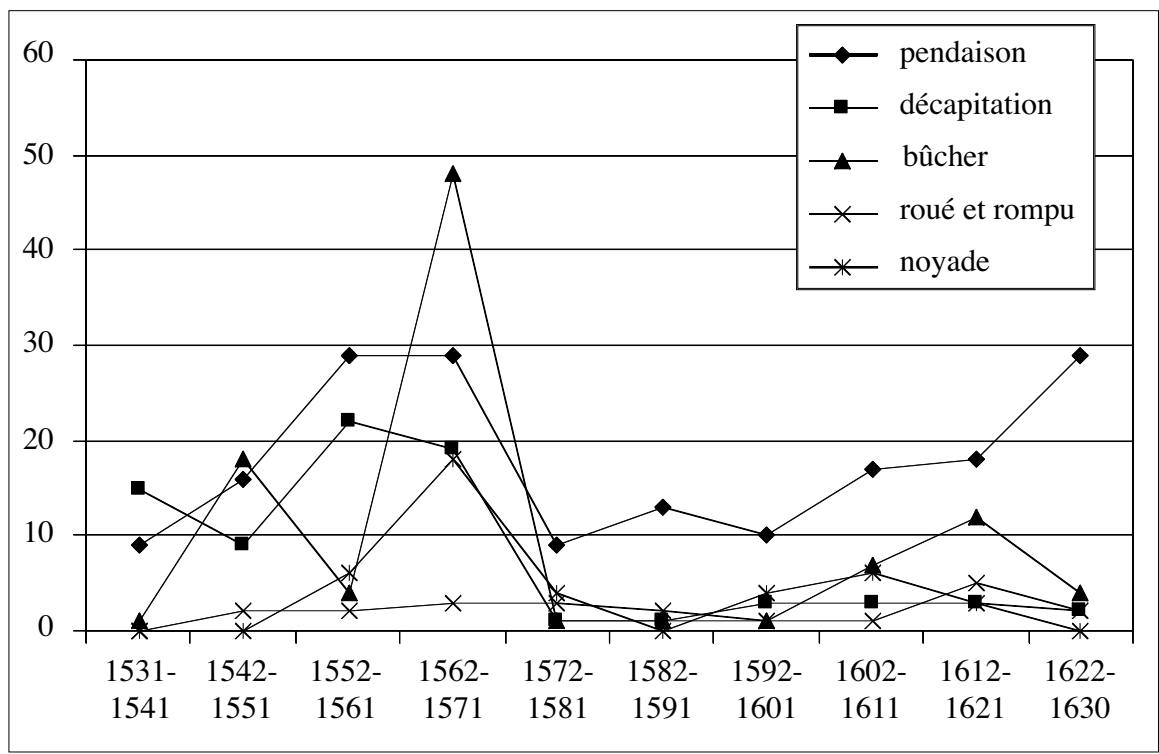

Figure 1: Nombre d'exécutions capitales par noyade et des autres modes d'exécution.

la noyade judiciaire rassemble des délits qui doivent être punis pour «donner exemple aux autres qui tel cas voudraient commettre ${ }^{8}$. Selon les juristes, auteurs des «Avis de droit» qui motivent la peine, il faut sanctionner «sans toutefois déclarer par menu [...] les moyens et circonstances desdites abominations afin d'éviter les inconvénients et plus grands scandales qui en pourraient advenir» ${ }^{9}$.

\section{NORMES \\ ET PRATIQUES DE LA NOYADE JUDICIAIRE}

De 1558 à $1575^{10}$, l'augmentation brutale du nombre d'exécutions capitales à Genève révèle l'intensification de la répression pénale. La pendaison est le plus souvent appliquée aux voleurs, la décapitation aux meurtriers ou aux faux-monnayeurs, la roue aux crimes composites et le bûcher à l'hérésie ou à la sorcellerie. De 1558 à 1575 , une exécution capitale sur sept est une noyade ${ }^{11}$ : une proportion révélatrice de l'intégration au système punitif genevois de ce nouveau mode d'exécution.

8 Formulation usuelle des sentences pénales genevoises. Pour une meilleure compréhension du texte je choisis de moderniser l'orthographe et la ponctuation des citations.

9 PC $8621^{\text {ère }}$ série, 1559, sodomie, avis de droit.

10 À partir de 1575 , le peu de fiabilité de l'inventaire des procédures ne permet pas de tirer des conclusions sur la proportionnalité entre le nombre de noyades et le nombre des exécutions.

11 De 1558 à 1575 , le nombre de noyades - qui je le rappelle sanctionnent l'infanticide, l'inceste, le viol d'enfants, l'adultère récidivant ou la sodomie - est de 23 pour un total de 157 exécutions capitales. 
Mode d'exécution:

"... estre noyé et submergé, façon accoustumé..."

Au Moyen-Âge, en immergeant l'accusé lors des jugements par ordalie des procès en sorcellerie, les juges recherchent l'obtention de la «preuve divine » pour décider de la culpabilité du prévenu ${ }^{12}$.

$\mathrm{Au} \mathrm{XVI}^{\mathrm{e}}$ siècle à Genève, la mort par l'eau est une pratique codifiée et ritualisée; elle ne constitue en aucun cas une étape de la procédure pouvant décider de la culpabilité de l'accusé. L'eau est un instrument de l'exécution au même titre que la roue ou la corde et la noyade clôt une procédure de type inquisitoire instruite par le Lieutenant de justice. À l'issue d'un procès à l'«extraordinaire» et après lecture publique de la sentence devant l'Hôtel de Ville, le condamné est «lié, attaché et traîné sur une claie» jusqu'au lieu d'exécution. En 1558, la première noyade répertoriée prend place «en l'eau du fleuve du Rosne» à l'extérieur de l'une des portes de la ville. Par la suite, s'adaptant aux modifications urbaines effectuées pour améliorer les fortifications de la cité, les exécutions prennent place au port du Molard puis au port de Longemalle, deux des trois débarcadères de la ville situés sur les rives du lac. L'iconographie judiciaire de la noyade en Suisse orientale illustre le spectacle offert à la population lors de l'exécution ${ }^{13}$ : les poignets et les chevilles liés ensemble, recroquevillé sur lui-même, le condamné est jeté depuis une barque, puis maintenu sous l'eau jusqu'à «tant que l'âme soit séparée de son corps». Alors que le Lieutenant de justice se tient sur la rive, l'exécuteur de haute justice ainsi que les «ministres du culte» chargés d'assister le condamné et de recueillir ses ultimes paroles se tiennent sur la barque, attendant sa mort. Dans la cité genevoise, à l'exception des recommandations du Conseil en 1558 et du témoignage d'un soldat en 1603 , il n'existe pas de description de la noyade judiciaire. Le témoin rapporte qu'une dispute éclate entre un Zurichois et un Français, tous deux réunis sur la même barque pour observer la noyade d'une femme condamnée pour infanticide ${ }^{14}$. Pendant ce temps, rassemblés autour du port de Longemalle, les Genevois se tiennent sur la jetée ou prennent place dans des embarcations pour assister au spectacle de l'exécution.

Le supplice s'achève par la mort du condamné dont le corps est ramené sur la terre ferme et, en ultime marque d'infamie, attaché sur une claie puis traîné jusqu'au gibet de la ville sous lequel il est enterré.

\section{Typologie des condamnés: "... lequel ayant oublié toute crainte de Dieu..."}

De 1530 à 1630, à Genève et dans le reste de l'Europe, la peine capitale s'applique plus fréquemment aux hommes qu'aux femmes - seulement $27 \%$ des condamnés à mort sont de sexe féminin. Pourtant, contredisant ces chiffres, le nombre total d'exécutions capitales par noyade se répartit de manière égale entre les deux sexes. Le Code criminel de la Caroline en vigueur dans le Saint Empire propose la noyade comme moyen d'adoucir la condamnation à mort sur le bûcher à

\footnotetext{
12 Mandrou (1980, pp. 182-183).

13 Schilling (1513).

14 Santschi (1974, p. 253).
} 
l'encontre des femmes ${ }^{15}$. Cependant, pour les magistrats genevois, le sexe de l'inculpé ne représente pas l'unique motivation pour condamner à la noyade: la qualification du crime, les circonstances des aveux ou la récidive influencent aussi la décision des juges.

«Sans avoir égard a ce qu'il a femme et enfants », «comme déjà par [là] même en l'âge de vieillesse »: les «sommaires» des procès rédigés à l'attention des juges, mentionnent le régime matrimonial et l'âge des condamnés. Le statut marital différencie la relation adultère de la paillardise, mais il peut aussi influer sur le choix de la peine lors d'un jugement en sodomie. Qu'ils soient hommes ou femmes, les inculpés dont la filiation est établie par le nom du père et précisée par le nom de l'époux pour les femmes, appartiennent à la communauté des justiciables. Leur métier ainsi que leur lieu de naissance sont aussi des critères d'identification. Cardeur, crocheteur, affineur: les hommes travaillent en tant qu'artisans ou domestiques, les femmes sont servantes ou épouses et mères de familles. Aucun des prévenus n'est de passage dans la cité, pas de vagabonds ni de voyageurs parmi ces «criminels».

D'un point de vue quantitatif, quarante pour cent des prévenus ont obtenu l'autorisation de vivre dans la cité genevoise en tant qu'Habitant, et ne possèdent pas de droits politiques ${ }^{16}$; vingt pour cent des condamnés possèdent le statut de Citoyen ou de Bourgeois; les quarante pour cent restants ont un statut indéfini, mais le lieu de leur naissance indique une origine française qui laisse à penser qu'ils sont issus du Refuge.

Dès 1550, suite aux répressions religieuses européennes, la cité de Calvin accueille un grand nombre de réfugiés étrangers qui acquièrent rapidement le statut d'Habitant ${ }^{17}$. L'augmentation de la population crée des tensions qui se reflètent à travers les différends politiques entre Genevois de souches et immigrés ${ }^{18}$. La concentration urbaine et l'exiguïté des logements facilitent le contrôle réciproque des individus soumis à une trop grande promiscuité. S'y ajoute la présence de dizeniers, et des ministres responsables du respect des règles et des ordonnances de la ville qui exercent une surveillance morale sur les membres de la communauté urbaine et religieuse. En s'installant librement dans la cité, les nouveaux arrivants ont adhéré obligatoirement à la Réforme et promis de se conformer à ses principes, ce qui leur est fermement rappelé en Consistoire lorsqu'ils fautent. Les instances ecclésiastiques n'hésitent pas à déférer les accusés devant les magistrats si elles estiment que la gravité du délit dépasse leurs compétences. Lors de procès pour transgressions sexuelles aggravées, il s'avère que les coupables ont souvent été réprimandés à plusieurs reprises par le Consistoire avant d'être déférés devant les juges du Petit Conseil ${ }^{19}$.

Les troubles politiques engendrés par la présence d'un grand nombre de riches étrangers qui cherchent à s'implanter dans les institutions genevoises sont souvent mentionnés dans les Registres du Conseil. En contrepartie, l'impact de la promis-

15 Punition des femmes dans le Code criminel dit de La Caroline, articles CXXXI, CXXXIII, CXXIV, CXXX, CLIX, CLXII.

16 Les droits politiques sont réservés aux Citoyens et aux Bourgeois. Pour plus d'informations concernant les différents statuts civils à Genève, voir Roth Lochner (1997).

17 Geisendorf (1957); Covelle (1897).

18 Grosse (1995); Mottu-Weber (2000).

19 PC $9571^{\text {ère }}$ série, 1561, Thomas de Reancourt, sommaire; PC $10611^{\text {ère }}$ série, 1562, Martin Leschières, sommaire. 
cuité sur la vie quotidienne de la population ainsi que l'animosité provoquée par l'afflux d'une population étrangère plus modeste n'apparaissent qu'en filigrane dans les procédures judiciaires et les dénonciations pour transgressions morales. Les crimes punis par noyade se caractérisent par une absence de témoignages à charge. Dans les cas d'infanticides ou de viols d'enfants, les victimes sont décédées ou trop jeunes, alors que durant les procès pour adultère, inceste et sodomie, les partenaires sexuels des accusés ne sont pas considérés comme des victimes de l'accusé par les magistrats et ils sont souvent poursuivis et condamnés. Ainsi la saisie par la justice est la conséquence de dénonciations de la part du voisinage et du contrôle social exercé par chacun ${ }^{20}$ plutôt que d'une plainte individuelle.

\section{Contentieux criminel: "... cas et Crime méritant griesve punition corporelle ..."}

Le renforcement $\mathrm{du}$ «contrôle moral» préconisé par Calvin s'inscrit dans un mouvement européen de durcissement punitif contre les «délits de mœurs ${ }^{21}$. S'inspirant du droit romain et des droits coutumiers locaux, les juristes genevois font preuve d'une sévérité accrue envers des crimes tels que la paillardise ou l'adultère $^{22}$. De 1558 à 1619 , une quarantaine de condamnés poursuivis pour infanticide, récidive d'adultère, inceste, viol d'enfant ou sodomie sont exécutés par noyade: les magistrats considèrent ces crimes comme des plus «atroces et abominables » et les assimilent à un comportement « contre nature».

En 1558, le Petit Conseil condamne Marie Bioran accusée d'avoir fait périr son nouveau-né:

Marie Bioran, détenue - [...] attendu qu'elle est [accusée] de larcins et d'avoir meurtri son propre enfant [...], Qu'elle soit traînée sur une claie jusque vers le lac et soit noyée pour donner exemple aux autres $[\ldots]^{23}$.

L'infanticide qui désigne le meurtre de l'enfant nouveau-né «fruit de la paillardise ou de l'adultère », est un crime souvent accompli pour dissimuler une grossesse illégitime. Durant toute la période médiévale en Europe - à l'exception de l'Angleterre $^{24}$ - l'infanticide est puni capitalement et considéré comme un péché et un acte contre-nature. Dès le milieu du XV $\mathrm{XV}^{\mathrm{e}}$ siècle, à la suite du durcissement pénal contre les délits moraux, l'infanticide et l'avortement sont désormais qualifiés aussi gravement que la sorcellerie ou l'hérésie ${ }^{25}$. En 1480, 1556 et 1586, en France, les Édits royaux témoignent de cette sévérité et condamnent la mère criminelle au bûcher. En 1532 dans le Saint Empire germanique, un article de la Caroline propose d'enterrer vivante ou de noyer la coupable ${ }^{26}$. À Genève, on ne retrouve aucune condamnation

\footnotetext{
20 Hoffmann (2004).

21 Carbasse (2000, p. 310).

22 Des criées règlementent la prostitution dans la cité en 1503 et 1513 ( $c f$. Sources du droit du canton de Genève, tome 2). Le durcissement de la surveillance morale apparaît dans l'Édit contre la paillardise et adultère de 1566 dont il est fait mention ultérieurement.

23 R.C. 54, fol. 290.

24 Dean (2001, p. 79).

25 Wrightson (1982).

26 Code criminel de l'empereur Charles V (1734).
} 
pour infanticide avant 1558 mais, à partir de cette date et jusqu'en 1619, les exécutions se pratiquent par noyade puis, dès 1623 , par pendaison.

Anne Lemoine et son serviteur Antoine Cossonnex, coupables du crime d'adultère aggravé par le lien domestique qui les unit, sont tout deux condamnés à la noyade:

\section{Mardi 13 août 1560}

Anne Lemoine femme de Pierre Bennard, Antoine Cossonnex son serviteur, pour avoir longuement adulteré ensemble [...] et elle d'avoir proféré plusieurs blasphèmes exécrables et lui aussi d'avoir voulu prendre par force Esther, la fille de ladite Anne comme ils ont confessé [...] qu'ils soient condamnés demain publiquement, elle a être noyée et lui avoir la tête coupée pour donner exemple aux autres $^{27}$.

Résolu par le biais d'une justice compensatrice et pécuniaire au Moyen-Âge, l'adultère est qualifié plus durement au $\mathrm{XVI}^{\mathrm{e}}$ siècle. En effet, sous l'influence du droit romain, les craintes pour l'héritage patrimonial en cas de filiation douteuse incitent à une plus grande sévérité envers l'adultère féminin. La femme adultère est fouettée et «authentiquée» mais n'est pas condamnée à mor ${ }^{28}$. À Genève, la répression culmine en 1566 avec l'acceptation en Conseil Général ${ }^{29}$ de l'Édit contre les paillardises et adultères. Ce texte qui semble s'inspirer du droit canon - lex de mulieribus et viris (la même loi pour les hommes que pour les femmes) - considère l'homme adultère aussi coupable que la femme. Se référant aux lois «divines et humaines », les magistrats qualifient l'adultère de crime capital et condamnent à la peine de mort aussi bien l'homme que la femme s'ils sont mariés. Bien que l'édit ne précise pas le mode d'exécution, en pratique, de 1560 jusqu'au début du XVII ${ }^{\mathrm{e}}$ siècle la noyade est appliquée aux femmes coupables d'adultère récidivant.

Toujours selon l'Edit de 1566, «L'inceste [est entendu] entre les personnes dont le mariage est prohibé et défendu simplement de la loy de Dieu et de nature ${ }^{30}$, ce que confirme la sentence de noyade prononcée à l'encontre de Jaques Saint Mortier et Andrée Viste, beau-frère et belle-sœur par alliance:

Nous conste et apert qu'ayant par la parole de Dieu en laquelle vous avez été dès longtemps [...] connu la grandeur et énormité du crime d'inceste [...] Toi Jaques Saint Mortier et toi Andrée Viste condamnons à devoir être liés de cordes et menés sur le fleuve du Rosne près la Corraterie et la en icelui être noyés et submergés façon accoutumée ${ }^{31}$.

Adultère entre une belle-mère et son gendre, relations illicites entre frère et sœur ${ }^{32}$, l'inceste peut se commettre dans la famille «réelle» ou dans la famille «spirituelle $»^{33}$, et sa répression se fonde sur l'interdit biblique ${ }^{34}$.

\footnotetext{
27 PC $9281^{\text {ère }}$ série, Livre des sentences criminelles, Jur. Pén. A2.

28 Carbasse (2000, pp. 310-313).

29 Conseil souverain de la cité qui rassemble les habitants de Genève possédant des droits politiques (Citoyens et Bourgeois).

30 Édit de 1566 dans Rivoire (1933, p. 170).

31 PC $13231^{\text {ère }}$ série, 1565 , sentence.

32 PC $15161^{\text {ère }}$ série.

33 Vigarello (1998, p. 47)

34 Lévitique (20; 12-22) dans La Bible de Segond (1974).
} 
Sévèrement réprimé à travers toute l'Europe, le viol d'enfant est puni de mort dès la première occurrence ${ }^{35}$. «Horrible et détestable forfait», acte infâme et «contre nature», la lecture des procédures révèle l'horreur et l'incompréhension des juges envers ce crime:

Tu as osé commettre l'horrible et détestable crime de stupre, faisant tes efforts de violer une fille si jeune, hors d'âge et impuissante [...] pervertissant ainsi par ta débordée et maudite affection et concupiscence l'ordre de nature qui doit être de tous inviolable [...] Toi Martin Leschière condamnons à devoir être lié et mené hors la porte de la corraterie et là être noyé et submergé en l'eau du fleuve du Rosne ${ }^{36}$.

Dès 1532 , le code de la Caroline en appelle au glaive à l'encontre des violeurs d'enfants. À Genève, de 1545 à 1559 , ils sont décapités, noyés à partir de 1562 puis pendus après 1580 .

En Europe, au $\mathrm{XVI}^{\mathrm{e}}$ siècle, le terme de sodomie désigne une relation sexuelle anale entre deux hétérosexuels, mais il englobe également la masturbation ${ }^{37}$, les relations homosexuelles masculines ou féminines, le viol d'un jeune garçon impubère ou encore la «bestialité $»^{38}$. Les juristes rassemblent ainsi sous l'appellation commune de sodomie tout acte sexuel sans finalité procréatrice. Considérée comme un acte contre nature, «la sodomie est d'abord crime moral: blasphème, transgression des lois divines ${ }^{39}$. Dès le XIII ${ }^{\mathrm{e}}$ siècle Thomas d'Aquin renforce l'interdit biblique et associe «crime de sodomie » et «atteinte à la nature de l'espèce ${ }^{40}$. Suite aux procès en hérésie contre les cathares, aussi appelés bogomiles et accusés de pratiquer la sodomie, le terme de «bougrerie » ou «bogrerie » s'avère mieux connu par la population que celui de sodomie, mot «savant» utilisé dans les procédures écrites: ce malentendu terminologique engendre parfois des quiproquos entre magistrats et accusés. La pratique de la sodomie est assimilée à une maladie transmise à l'accusé dans sa jeunesse après qu'il ait été lui-même «sodomisé». L'analogie entre comportement criminel et mal contagieux provoque la peur d'une contamination de l'ensemble du corps social de la communauté. Seule une punition exemplaire et sévère peut endiguer le phénomène de propagation. À une époque marquée par les grandes épidémies de peste, cette représentation entre parfaitement en résonnance avec les récits bibliques de Sodome et Gomorrhe, récits auxquels les «Avis de droit» font souvent référence lors des procès pour sodomie. À Genève, extraire le péché pour éviter la vengeance de Dieu est une évidence pour les magistrats et de 1561 à 1614 , les «sodomites » sont systématiquement noyés ou menés au bûcher lorsqu'il s'agit d'étrangers ${ }^{41}$ :

35 Il s'agit essentiellement de viols de fillettes non pubères, les viols de garçons en dessous de l'âge de la puberté sont considérés comme des actes de sodomie.

36 PC $10611^{\text {ère }}$ série, 1562, Martin Leshière.

37 Davidson (2002, p. 66).

38 C'est-à-dire les relations sexuelles entre un homme et un animal.

39 Vigarello (1998, p. 41).

40 Puff (2004).

41 RC 73,f. 127, Marco Colnio, 1578; PC $2^{\text {ème }}$ série, 1634, 1590. 
Jeudi XIII novembre 1600, Pierre Dufour, Pierre Brelat, détenus pour avoir commis ensemble le crime de sodomie, arrêtés que soient condamnés tous deux à être noyés lundi prochain ${ }^{42}$.

Ainsi, au $\mathrm{XVI}^{\mathrm{e}}$ siècle, les transgressions sexuelles et les crimes «contre nature» sont réprimés tout aussi sévèrement à Genève que dans le reste de l'Europe ${ }^{43}$. Mais de 1558 à 1619, particularisme de la République du bout du lac, la peine capitale par noyade est le mode d'exécution privilégié à l'encontre de ce contentieux criminel.

\section{CHOIX D'UN NOUVEAU MODE D'EXÉCUTION}

\section{Contexte politico-religieux}

En 1526, Genève rompt ses liens de dépendance avec la Savoie et en 1536 les Citoyens et les Bourgeois de la ville acceptent librement d'adhérer à la Réforme. À partir de 1541, Calvin, en réponse à l'appel des autorités civiles, s'installe à Genève durablement et tente d'appliquer les principes de la nouvelle religion ${ }^{44}$. Ces changements qui provoquent des répercussions sur le fonctionnement des juridictions aussi bien ecclésiastiques que civiles, affectent par conséquent la vie quotidienne des habitants de la cité. De 1541 à 1555 , Calvin et les ministres réformés s'opposent fréquemment aux magistrats du Petit Conseil, l'instance exécutive de la ville. Les désaccords se manifestent tout particulièrement au sujet du contrôle moral et de la légitimité du droit de sanction du Consistoire, organe de contrôle ecclésiastique et moral mis en place dès 1541 . En fait, cette situation conflictuelle alimente les différends politiques entre les Genevois de souche et les Français récemment réfugiés qui s'implantent progressivement au sein des institutions civiles. En 1555, la lutte d'influence entre les deux partis débouche sur la victoire des partisans de Calvin. Au cours des années suivantes, à Genève comme dans le reste de l'Europe, la répression envers les crimes moraux se radicalise. Bien que la poursuite et la sanction des comportements délictueux tels que le blasphème ou la paillardise dépendent du Consistoire, les coupables sont déférés aux mains des magistrats et jugés en criminels si la juridiction ecclésiastique considère les crimes comme trop graves. En 1558, dans ce contexte politico-religieux, l'exécution capitale par noyade fait son apparition dans la République genevoise.

Selon P. Spierenburg, à partir du XII ${ }^{\mathrm{e}}$ siècle et jusqu'au XVI $\mathrm{X}^{\mathrm{e}}$ siècle, à Amsterdam, le durcissement de la justice criminelle et l'augmentation des peines afflictives et capitales reflètent la consolidation des autorités urbaines: la justice pénale vise le crime et son impact sur la communaute ${ }^{45}$.

À l'instar du modèle hollandais, il semble que les instances exécutives de Genève affermissent leur autorité par un renforcement pénal. Ce phénomène de durcissement de la répression se développe en même temps que les changements institutionnels

\footnotetext{
42 PC $18181^{\text {ère }}$ série, 1600, et RC 95.

43 À l'exception de la répression de l'adultère qui occupe une place particulière à Genève suite à la pression exercée par les ministres réformés.

44 Et plus précisément, les principes contenus dans les Ordonnances ecclésiastiques de la ville de Genève de 1541.

45 Spierenburg (1984, pp. 5-12).
} 
genevois et apparaît particulièrement sévère à l'encontre des crimes «moraux». S'ajoute à ces modifications l'impact de la dimension religieuse spécifique à Genève. Bien que les champs d'application des justices ecclésiastique et pénale se distinguent par les délits poursuivis et les peines appliquées, les autorités civiles fonctionnent en lien étroit avec les autorités ecclésiastiques plus particulièrement après 1555 . Pour Calvin, à la fois théologien et juriste, l'exercice de la justice dépend d'une autorité politique sécularisée ${ }^{46}$. Il existe un sens théologique de la peine et du droit de punir, mais le magistrat reste «l'intercesseur» entre justice divine et société. Les idées de Calvin sur la «loi divine» et sur la prédestination de l'individu s'accordent aux caractères traditionnels de rétribution et d'exemplarité de la peine. "Versions affaiblies de la loi divine, les "lois civiles" délimitent un champ plus restreint de délits » qui s'inscrivent à la fois, contre la loi de Dieu et contre la loi de la sociétét ${ }^{47}$. De plus, malgré le principe de prédestination, l'homme, responsable de sa faute, mérite une punition afflictive et exemplaire. L'acte mauvais ne pouvant venir de Dieu, il faut le sanctionner; la justice divine doit être appliquée par les autorités humaines pour éviter que la vengeance divine ne s'exerce sur l'ensemble de la communauté.

Malgré des principes clairement énoncés par Calvin sur la séparation des rôles entre justice ecclésiastique et civile, la répartition des fonctions au sein des institutions révèle les interférences et l'imbrication des liens entre Consistoire et Petit Conseil. Les ministres réformés de la cité et douze Anciens dont trois membres du Petit Conseil forment le Consistoire. De par leurs charges, certains magistrats sont donc juges au tribunal pénal tout en étant responsables de la discipline ecclésiastique. Théodore de Bèze mène personnellement certains interrogatoires criminels et parfois les juges et les modérateurs de la Compagnie des Pasteurs n'hésitent pas à «conférer» sur l'opportunité d'infliger la peine de mort ${ }^{48}$. Les livres des sentences criminelles signalent à plusieurs reprises que des avis doivent être demandés à $«$ M. Calvin $»^{49}$ ou $«$ M. de Bèze $»^{50}$, bien qu'il ne subsiste que des avis de droits signés de Colladon dans les dossiers de procédures correspondants.

À Genève, l'influence réciproque entre État républicain et Église réformée s'exprime à travers les liens personnels et les conflits d'autorités et singularise ainsi la culture judiciaire de la République.

\section{"... Requérir l'avis des 'Advocats...'»}

Dès le milieu du XVI ${ }^{\mathrm{e}}$ siècle, confrontés à des crimes et des «choses non jamais ouïes», les magistrats du Petit Conseil recourent aux «avis des advocats $»^{51}$. Pratique héritée des commentateurs du droit romaniste, la rédaction des «Avis de droit» témoigne des questionnements et des hésitations des juges. Qualifier le crime, réglementer la procédure et motiver la peine, ces «avis» éclairent la pratique pénale:

\footnotetext{
46 Carbonnier (1974, pp.187-201).

47 Carbonnier (1974, p. 190).

48 PC $17131^{\text {ère }}$ série, 1581, Suzanne Fontana.

49 Jur. Pén. A3, vendredi 28 août 1562, fol. 58 v. et lundi 31 août, fol. 59 v., Martin Leshière.

50 Jur. Pén. A4, mardi 8 mai 1565, fol. 10 v., Pierre Mibor et Pierre Gardet; Jur. Pén. A4, 12 décembre 1565, fol. 82 v., Jaquema Masson; Jur. Pén. A5, vendredi 26 mars 1568, fol. 15, Françoise Mazel.

$51 \quad$ PC $9281^{\text {ère }}$ série, 1560 et PC $9451^{\text {ère }}$ série, 1560.
} 
Et que icelluy crime est des plus atroces et abominables que l'on trouve point comme appert par l'écriture sainte et par le droit aussi c'est une chose trop étrange et monstrueuse en telle jeunesse ${ }^{52}$.

Leurs auteurs sont des juristes réformés et des convertis de la première heure qui puisent leur inspiration chez les commentateurs du jus romanum du XIV ${ }^{\mathrm{e}}$ siècle tels que Balde ou Bartole mais aussi dans les versets de la Bible et plus particulièrement dans le Décalogue ou le Deutéronome. Occasionnellement un édit local ${ }^{53}$ ou la coutume servent à justifier les arguments avancés par les juristes. Ces références sont inscrites et annotées dans les marges des «Avis de droit». Parmi les auteurs des avis, Germain Colladon (1508-1594) ${ }^{54}$, proche de Calvin et de Théodore de Bèze, émet 18 des 23 avis concernant les noyades judiciaires. D'autres juristes, tels que François Chevallier ${ }^{55}$ ou Laurent de Normendie ${ }^{56}$ sont aussi sollicités lors des procès pour crimes «contre nature».

\section{Motiver la peine}

Proposer une sentence de noyade judiciaire résulte d'une réflexion des juristes sur la qualification du crime et la motivation de la peine. Dès 1554 , cinq garçons, dont deux impubères qui par leur «trop grande jeunesse, $[. .$.$] n'ont pu appréhender$ [...] l'horreur et atrocité dudit crime» et trois au-dessus de l'âge de puberté, dotés de «jugement» et possédant «capacité de raison », sont poursuivis pour avoir commis le «crime» de sodomie. Les plus jeunes sont exemptés de peine capitale en raison de leur âge, quant aux trois autres:

Ils ne méritent la mort toutefois non pas tant rigoureuse que si lesdits garçons eussent été plus âgés et suffirait à rigueur de droit pour rendre la peine correspondante à tel crime et moins rigoureuse pour leur jeunesse qu'ils fussent noyés ${ }^{57}$.

Pour la première fois les juristes suggèrent le recours à la noyade pour adoucir la condamnation à mort sur le bûcher, peine capitale habituellement appliquée à l'encontre des «sodomites $»^{58}$. En 1558, Marie Bioran est convaincue d'infanticide: «ledit crime a été trouvé si énorme et exécrable» que Germain Colladon remonte aux origines du droit romain et se réfère à l'application du supplice du culleus $^{59}$. Dans son «avis », jugeant la peine obsolète, il modère la peine du feu par celle de la noyade, en raison des aveux de la prévenue:

52 PC $5021^{\text {ère }}$ série, 1554, avis de droit.

53 Les Édits de 1566 dans le PC $15941^{\text {ère }}$ série, 1570, adultère récidivant.

54 Germain Colladon est originaire du Berry. Il reçoit une formation de légiste à Bourges puis à Orléans. En 1550, il émigre à Genève dont il acquiert la bourgeoisie en 1555. Il participe à la vie de la cité jusqu'à sa mort; Kaden (1974).

55 Juriste et magistrat. Sa famille acquiert la bourgeoisie genevoise dès 1506.

56 Réfugié à Genève en 1548, docteur en droit, Laurent de Normendie pratique comme juriste avant de devenir éditeur (?-1569).

57 PC $5021^{1 \text { ère }}$ série, 1554, 5 garçons anonymes, avis de droit (je souligne).

58 Évaluation faite en fonction des documents subsistants. Finalement, la peine capitale n'est pas appliquée aux jeunes garçons qui seront bannis à vie.

59 Il s'agit d'une peine réservée au parricide, qui consiste à enfermer le condamné dans un sac avec un singe, un coq, un chien, une vipère avant de le jeter à l'eau. 
Toutefois telle peine ne se pratique aujourd'hui mais la peine du feu est en plus commun usage. Comme aussi la confession spontanée de ladite Marie doit mitiguer ladite peine $[\ldots]$ y aura nature pour la condamner à être noyée après avoir été traînée sur une claie par la ville et jusque à l'eau et lieu du supplice $[\ldots]^{60}$.

L'exécution de Marie Bioran représente le premier et unique cas de noyade judiciaire à l'occasion duquel il est fait état de précisions techniques, telles que l'usage d'une barque ou le choix du lieu d'exécution ${ }^{61}$ : détails révélateurs de la nouveauté de l'application de cette peine. En 1559, Jaquema Gonet qui a commis «les actes abominables de sodomie, de péché contre nature » avec la jeune fille de la maison où elle travaille, est aussi condamnée à être noyée:

À ces causes ne peut être exemptée de peine de mort exemplaire comme d'être noyée et suffoquée en l'eau car si l'on voulait la punir à la rigueur de droit elle serait condamnée à être brûlée ${ }^{62}$.

La «rigueur de droit» indique le degré de sévérité que devrait atteindre la sanction en fonction de la gravité du crime. Les juristes conseillent cependant la mansuétude: l'eau tout en adoucissant la cruauté de l'exécution, permet de conserver le caractère d'exemplarité de la peine capitale. En 1561, Thomas de Réancourt a tenté de commettre «actes de sodomie [...] choses tant vilaines et exécrables et d'une continuation tant débordés qu'il n'est possible de l'excuser ni exempter de peine de mort», il s'ensuit que:

Combien qu'il n'ait du tout accompli le crime de sodomie en sa propre espèce, ce qui l'exempte de la griesve peine du feu toutefois [...] il doit être condamné à la mort et par ce que n'est un crime non accoutumé sera bon que la peine de mort soit spéciale $[\ldots]$ à savoir d'être noyé ${ }^{63}$.

La même année, Guillaume Branlard est aussi accusé de sodomie:

Par quoi il est digne de mort, toutefois à cause de la spontanée confession la peine rigoureuse du feu pourra être commuée à moindre peine [...] d'être noyé et mourir en l'eau ${ }^{64}$.

De 1554 à 1564, malgré le durcissement général de la répression à l'encontre des crimes «contre nature» en Europe, les juristes genevois trouvent des arguments qui les entraînent à prescrire une peine «adoucie». Sans citer leurs sources d'inspiration ni leurs modèles juridiques, ils considèrent l'exécution par noyade comme un adoucissement de la peine de mort par le feu. Les raisons avancées en faveur d'une «mitigation» de la peine retiennent des caractéristiques propres à l'individu telles que la jeunesse ou le sexe. Dans d'autres cas, ils prennent en considération les aveux spontanés ou l'acte criminel partiellement commis. Bien que la peine s'adresse au crime et non

\footnotetext{
60 PC $7561^{\text {ère }}$ série, 1558, Marie Biorant, infanticide, avis de droit.

61 RC 54, 1558, 2 septembre; 6 septembre; 19 septembre; 20 septembre; 22 septembre.

62 PC $8621^{\text {ère }}$ série, 1559, Jaquema Gonet, sodomie, avis de droit.

63 PC $9571^{\text {ère }}$ série, 1561, Thomas de Réancourt, sodomie, avis de droit.

64 PC $9711^{\text {ère }}$ série, 1561, Guillaume Branlard, sodomie, avis de droit.
} 
au criminel ${ }^{65}$, à Genève, les juges et les juristes considèrent le comportement de l'accusé. Faut-il voir dans cette attitude une influence du Code criminel de la Caroline de 1532 ou celui des Sources du droit bernois? Ces textes préconisent «que les femmes condamnées à mort ne dussent être mises au gibet, mais être noyées et jetées en la rivière aux lieux là où l'on trouve des rivières ${ }^{66}$. Si les juristes ne citent pas leurs sources d'inspiration pour appliquer une peine «mitigée », ils se singularisent toutefois en exécutant par noyade aussi bien les hommes que les femmes. Le sexe n'est plus l'unique motivation pour adoucir la peine, s'y ajoutent la jeunesse de l'accusé ou la spontanéité des aveux qui sont aussi des critères d'atténuation du supplice infligé.

De 1564 à 1566, dans leurs «Avis de droit», les juristes se réfèrent à des condamnations antérieures prononcées à l'encontre de crimes similaires et ils ne motivent plus le choix de la noyade comme mode d'exécution:

Par quoi semble qu'elle doit être condamnée à la peine de mort accoutumée en tel cas à savoir d'este noyée et finir sa vie en l'eau et son corps être après mené au lieu de Champel ${ }^{67}$.

Il suffit de se référer aux peines «accoutumée» ou aux «lois impériales» pour condamner à la noyade sans que l'âge ou la spontanéité des aveux ne soient pris en compte. Pourtant, occasionnellement, il ressort que la peine capitale par l'eau continue à être considérée comme un «adoucissement» de la peine du feu:

lequel cas fait détestable et contre nature mérite la peine de mort par le feu selon les lois impériales sinon qu'on veuille user de la peine de mourir par eau comme a été fait en d'autres $\operatorname{cas}^{68}$.

Puis à partir de 1566, la noyade cesse d'être considérée comme une «mort adoucie»:

Ledit Tecia ne peut être excusé de trop grande jeunesse et ignorance car il a eu recommencement et a plusieurs fois constamment nié ledit cas après la confrontation [...]. Et si du commencement il [eut] confessé et reconnu sa faute y eu heu quelques apparences de modérer la punition mais ledit cas étant tel que dessus par le droit et parole de dieu il est coupable dudit crime qui mérite la mort et en semblable a été condamné [...] un jeune compagnon imprimeur [...] et aussi une jeune garce chambrière qui fut noyée ${ }^{69}$.

L'absence d'aveux spontanés et la récidive de l'acte criminel empêchent les juristes d'invoquer le jeune âge de l'accusé, collégien au moment du «crime». Pourtant, la peine de référence reste la noyade, précédemment appliquée pour des actes de sodomie. La noyade judiciaire cesse-t-elle de représenter un adoucissement de la peine ou devient-elle une peine réservée à des transgressions sexuelles? De 1568 à 1619 , malgré plusieurs cas de mise à mort par noyade, les avis ne mentionnent plus

\footnotetext{
65 Muchembled (2006).

66 L'exécution des femmes condamnées à mort, dans Matzinger-Pfister (2003, p. 81) mais aussi les articles CXXXI, CXXXIII, CXXIV, CXXX, CLIX, CLXII, du Code criminel dit de La Caroline.

67 PC $11901^{\text {ère }}$ série, 1564, avis de droit, Marie Binot, adultère répété.

68 PC $14651^{\text {ère }}$ série, 1568, avis de droit, Françoise Mazel, sodomie.

69 PC $13591^{\text {ère }}$ série, 1566, Bartholomé Tecia, sodomie, avis de droit.
} 
les raisons du choix d'une telle peine. Pourtant Marco Colnio ${ }^{70}$ en 1578 et cinq prisonniers ( 3 esclaves turcs et 2 Français) en 1590 sont exécutés sur le bûcher pour crime de sodomie. L'exécution à mort par le feu appliquée à des étrangers à la Cité pourrait nous inciter à penser que l'exécution par l'eau demeure parfois une forme «mitiguée » d'application de la peine capitale du bûcher destinée à marquer la différence entre le statut des Genevois et celui des étrangers.

Les Registres du Conseil ne mentionnent pas la renonciation à ce type d'exécution mais la dernière occurrence de noyade judiciaire répertoriée en 1619 marque la fin d'un usage pénal à Genève. Bien que la condamnation au bûcher à l'égard des «sodomites » perdure jusqu' au milieu du XVII ${ }^{\mathrm{e}}$ siècle, et que les mères infanticides soient encore exécutées au XVIII ${ }^{e}$, d'autres crimes tels que l'adultère voient leur répression s'adoucir à partir du XVII ${ }^{\text {e }}$ L'interruption de l'application d'un mode d'exécution tel que la noyade judiciaire témoignerait alors d'un changement de la qualification de certains crimes et d'un abandon progressif de la diversification des peines.

\section{Grammaire visuelle de la peine}

Au delà des écrits et des arguments avancés par les juristes, d'autres éléments justifient l'apparition d'un nouveau mode d'exécution comme la noyade. À partir des années 1560-1562, il semble qu'un processus se mette en place pour tenter de souder publiquement sur la scène punitive (échafaud ou barque), la nature du crime au régime suppliciaire de la peine. À la publicité et à l'exemplarité du supplice s'ajoute la fonction de «cérémonie de l'information» entre l'État et la population ${ }^{71}$. Ce dialogue se manifeste par un code qui emploie des peines diversifiées et spécifiques comme autant de «miroirs» des crimes commis. «L'exécution publique des criminels n'est pas seulement un phénomène punitif. Elle constitue aussi l'une des formes les plus spectaculaires de communication entre le pouvoir et les sujets [...], d'importantes modifications du rituel prennent place dès la fin du Moyen-Âge, signe de profonde évolution du dialogue muet que la justice noue à chaque occasion avec les spectateurs $»^{72}$. Ainsi, le «spectacle de la souffrance» fonctionne en tant que mode de prévention générale du crime ${ }^{73}$. Spierenburg mentionne le recours à des peines diversifiées et spécifiques au crime, des «peines miroirs», reflets des crimes commis ${ }^{74}$. Ce phénomène s'exprime par exemple lors de la condamnation à un simulacre de décapitation: le bourreau agite une épée au-dessus de la tête du condamné accusé d'avoir «presque» tué un homme ${ }^{75}$. Sur ce modèle, l' «avis» émis en 1561 lors du procès de Thomas de Réancourt et qui n'est pas suivi par le Petit Conseil, suggère la noyade, peine capitale encourue normalement pour un crime de sodomie:

Combien qu'il n'ait du tout accompli le crime de sodomie en sa propre espèce ce qui l'exempte de la griesve peine du feu toutefois [...] il doit être condamné à la mort $[\ldots]$ à savoir d'être noyé et submergé en l'eau ${ }^{76}$.

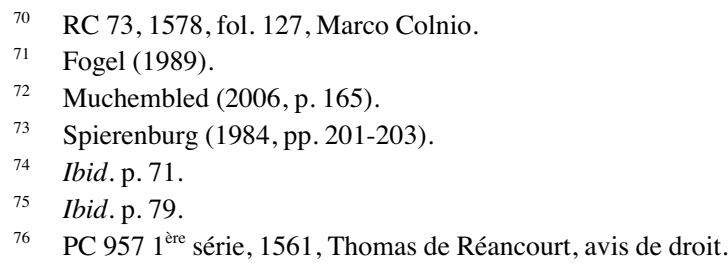


Finalement, le condamné est soumis à un simulacre de noyade qui épargne sa vie mais vise peut-être à le purifier:

Détenu pour avoir fait plusieurs vilains attouchements envers plusieurs et diverses personnes mâles tendant et approchant au crime de sodomie a été condamné à devoir être plongé trois fois en l'eau et puis banni perpétuellement à peine d'être entièrement submergé et noyế ${ }^{77}$.

La mise en pratique d'une forme d'analogie suppliciaire entre le crime commis et la peine infligée a pour conséquence une condamnation «partielle» à l'encontre de l'auteur du crime «inachevé». La noyade partielle appliquée à Thomas de Réancourt transforme la mise en pratique de l'exécution publique en un message de terreur préventive adressé au public autant qu'au criminel. La peine, «image symbolique du crime $»^{78}$, est associée au crime par analogie visuelle. En 1554, l' «Avis de droit » signé par plusieurs juristes et ministres dans le cas du procès de cinq jeunes garçons accusés de sodomie, illustre parfaitement la fonction d'analogie visuelle à visée pédagogique de la peine ${ }^{79}$. Les légistes conseillent des peines différentes à l'encontre des accusés les plus jeunes du fait de leur méconnaissance du crime. Ils leur prescrivent:

Qu'ils doivent être battus de verges en lieu secret et en la présence de nosdits seigneurs et aucun de leurs parents afin que lesdits jeunes garçons souffrant telle peine en la présence de leur dits parents aient à l'advenir crainte d'eux et se rendent plus sujets à leurs castigations ${ }^{80}$ et admonition et pour leur donner mieux à cognaistre la grâce qu'on leur a faite et l'abomination de leur mal sera bon de leur représenter la peine du feu en brûlant quelques fagots devant eux avec grand commination de ladite peine du feu s'ils y retournent ${ }^{81}$.

L'association visuelle fonctionne à deux niveaux: le châtiment du fouet, appliqué en présence des juges mais concomitant à la présence des proches, renforce de manière préventive l'autorité future des parents alors que la présence des fagots symbolise la peine réservée en cas de récidive d'un tel crime.

Tout comme la roue, le bûcher ou la pendaison, la noyade judiciaire s'intègre donc parfaitement dans l'espace punitif d'Ancien Régime à l'intérieur duquel le spectacle suppliciaire fonctionne comme un rappel du crime réprimé à l'attention de la population. Pourtant, une question persiste quant à la mise en application d'un nouveau supplice par l'eau. Nouveau lieu d'exécution, nouvelle pratique, l'exécution par noyade n'est pas improvisée: il s'agit bien d' «inaugurer» l'usage d'une peine particulière visant un type de délit spécifique. Les atteintes aux mœurs ainsi que les crimes de lèse-majesté appartiennent à «la sphère du sacré [ils] se situent au delà des limites du tolérable $»^{82}$. La noyade sanctionne des crimes dont les magistrats

77 PC $9571^{\text {ère }}$ série, 1561, Thomas de Réancourt, Livre des criminels, fol. 160v., Jeudy 10 juillet 1561.

78 Muchembled (2006).

79 Les cosignataires de cet avis sont Jean Calvin, Abel Poupin (ministre), Germain Colladon (juriste) et Françoys Chevallier (magistrat).

80 Avertissement, reproche, châtiment.

$81 \quad$ PC $5021^{\text {ère }}$ série, 1554, avis de droit.

82 Muchembled (2002, p. 226). 
craignent la dimension «contagieuse», ce sont des crimes «contre nature» que les juges ne décrivent qu'avec beaucoup de réticences:

De combien que lesdits cas et crimes soient tant abominables qu'il serait à désirer que telles personnes (coupables desquels crimes) fussent exterminées sans qu'il en fut jamais mémoire ni que le peuple n'entendisse ladite abomination. Toutefois puisque cela est révélé en justice et découvert selon qu'il plu à Dieu faut que la punition s'en ensuive sans toutefois déclarer par la même et trop [...] les moyens et circonstances desdites abominations afin d'éviter les inconvénients et plus grands scandales qui en pourraient advenir ${ }^{83}$.

Certains crimes ne devraient pas être nommés. Il faut éviter «que le peuple n'entendisse ladite abomination» et qu'ainsi il ne soit tenté de la reproduire. Dans le même temps, Dieu attend des juges une punition publique et exemplaire du crime dont il a permis la découverte. Ne pas le sanctionner ferait courir à la communauté toute entière le risque de la vengeance divine. Dissimuler le crime pour en éviter la propagation tout en rendant la punition exemplaire et donc publique, tel est le dilemme devant lequel se trouvent juristes et magistrats lorsqu'ils doivent punir certains crimes «moraux». Ce phénomène est clairement énoncé lors des procès pour sodomie ou bestialité :

Et y aurait plus grand danger et scandale en l'impunité ou couvert [...] de la peine qu'en la punition publique et exemplaire attendu aussi qu'il faut craindre l'ire de dieu qui n'a voulu remettre tel crime et en tant de personnes pour le laisser impuni mêmes en tel lieu. Et serait danger que notre seigneur manifesta avec plus horrible punition ce que les hommes auraient voulu cacher ${ }^{84}$.

Pour Calvin, le châtiment possède une fonction de prévention générale. Il effraie «les méchants» mais surtout, «il faut que l'horreur de la peine rejaillisse sur le crime, et qu'ainsi le crime soit en horreur [...]. La punition joue ici un rôle de symbole, de signifiant qui renvoie à un signifié. Il s'agit donc en quelque sorte d'un modelage de l'opinion publique. La punition doit frapper les esprits pour que l'acte puni paraisse rétrospectivement comme digne d'être puni, pour que le crime soit reconnu comme crime et abomination aux yeux de tous ${ }^{85}$. L'horreur de la peine fonctionne en analogie à l'horreur du crime. L'ampleur du crime doit être révélée sans description verbale et le crime «contre nature « «identifié » à la représentation visuelle de la peine appliquée. Le spectacle de l'exécution provoque aux yeux du public l'association entre une peine particulière et un crime indicible. La noyade, qui réserve à ses victimes une mort «non visible» puisque le condamné meurt sous la surface de l'eau, figure la mort cachée. Une mort dissimulée en réponse à un crime indicible qui doit être puni publiquement «afin de mieux ensuivre et représenter par l'espèce et la peine la détestation dudit crime ${ }^{86}$.

\footnotetext{
83 PC $8621^{\text {ère }}$ série, 1559, Jaquema Gonet, avis de droit.

84 PC $5021^{\text {ère }}$ série, 1554, avis de droit.

85 Carbonnier (1974, p. 195).

86 PC $7561^{\text {ère }}$ série, 1558, Marie Bioran, avis de droit.
} 


\section{CONCLUSION}

L'exécution capitale par noyade n'est pas une invention des systèmes punitifs européens du $\mathrm{XVI}^{\mathrm{e}}$ siècle. Qu'il ait été appliqué ou non, le supplice du culleus est un mode opératoire à l'encontre du parricide connu depuis l'Antiquité. Aussi lorsque Germain Colladon propose d' «enclourre en un grand sac de cuir avec plusieurs bêtes savoir un singe, un chien, un serpent nommé vipère et un coq et le tout ensemble jeter en l'eau pour le faire languir misérablement», il se démarque immédiatement en indiquant que «toutefois telle peine ne se pratique aujourd'hui mais la peine de feu est en plus commun usage $»^{87}$. En 1482, pour épargner l'honneur d'un condamné et à la requête de sa famille, l'évêque de Genève demande aux juges de commuer une peine publique en noyade clandestine ${ }^{88}$. La peine de mort par noyade est un «supplice considéré alors comme moins infamant que la pendaison pour la renommée de la famille du condamné ${ }^{89}$. Les considérations qui président à ce choix visent-elles à dissimuler la mise à mort de l'individu en transformant une peine publique en peine privée ou entend-on cacher le crime dont il est accusé?

Selon Clasen $^{90}$, lors de la répression des Anabaptistes en Suisse orientale, la noyade représente un usage local d'application de la peine capitale. Les crimes dont les Anabaptistes sont accusés diffèrent du contentieux criminel genevois de la noyade: chargés pour «hérésie» ou «rebellions» contre les lois civiles, ils ne sont jamais condamnés pour crime «contre nature».

À partir de 1558, à Genève, la pratique de la noyade judiciaire répond à une volonté de diversifier le catalogue des peines en vigueur. Tout au long de la mise en application de ce nouveau mode d'exécution, les motivations des juges et des juristes genevois évoluent. Dans un premier temps, la noyade est adaptée à l'individu accusé tout en sanctionnant le crime. Il s'agit d' «adoucir» la mise à mort pour les femmes, les jeunes ou les condamnés ayant avoué leur crime. Après une dizaine d'années d'application, la motivation du choix de la peine se modifie et les juristes considèrent le crime commis plutôt que l'individu. La «mitigation de la peine » n'est plus invoquée par les auteurs des «Avis de droit». La noyade devient un moyen d'identifier et de catégoriser visuellement plutôt que verbalement les crimes contre la «moralité ». Pour éviter la colère divine, les magistrats doivent punir des comportements intolérables. Ils en craignent la dissémination qui pourrait être favorisée par la description trop explicite du crime sexuel. L'analogie visuelle crée un lien entre le crime «indescriptible» et sa sanction. La dimension pédagogique de la peine ainsi respectée, les juges évitent de nommer des crimes qu'ils répugnent à décrire. La mort «dissimulée » par l'eau et le spectacle de la noyade concourent à formaliser le lien rétributif entre la publicité obligée de la peine et le scandale indicible du crime «amoral».

Dès 1620 , la condamnation à mort par noyade cesse d'être prononcée à Genève. L'arrêt de sa mise en application précède de quelques années seulement l'amorce d'une uniformisation du catalogue des peines capitales. À partir de 1630, le nombre d'occurrences des différents modes d'exécution capitale diminue. Bien que les

\footnotetext{
87 PC $7561^{\text {ère }}$ série, 1558, Marie Bioran, avis de droit.

88 Rivoire (1930).

89 Gauvard (2000, p. 100).

90 Clasen (1972, pp. 370-383)
} 
condamnations au bûcher persistent et que la roue soit encore utilisée occasionnellement, la pendaison devient le mode d'application de la peine capitale le plus fréquemment prescrit jusqu'à la fin de l'Ancien Régime.

\author{
Sonia Vernhes Rappaz \\ 11, place des Augustins \\ CH-1205 Genève \\ sonia.vr@bluewin.ch
}

\title{
CHOIX BIBLIOGRAPHIQUE
}

\section{Sources manuscrites}

Procédures Criminelles de Genève (= PC), Archives d'État de Genève (AEG).

Livre des sentences criminelles, AEG, Jur. Pén. A.

Registres du Conseil (= RC), AEG.

\section{Sources imprimées}

Matzinger-Pfister, R., Les Sources du droit Suisse - canton de Vaud, tome C, Bâle, Schwabe, 2003, p. 81.

Rivoire, É. (éd.), Sources du droit du Canton de Genève, vol. 2 (1461-1550), Aarau, H.R. Sauerländer, 1930.

Rivoire, É. (éd.), Sources du droit du Canton de Genève, vol. 3 (1551-1620), Aarau, H.R. Sauerländer, 1933.

THESAURUS EPISTOLICUS CALVINIANUS, Calvini Opera, vol. XV, Brunsvigae, 1876.

«Drowning by throwing from the bridge, Constance, 1486 » et «Drowning of Pete Wunderlich in Zurich» in Lucerne Chronicles by Schilling, D., 1513.

Code criminel de l'empereur Charles V, vulgairement appelé la Caroline, Paris, 1734.

Segond, L. (trad.), La Sainte Bible, Paris, La Maison de la Bible, 1974.

\section{Références}

Blondel, L., Le développement urbain de Genève à travers les siècles, Cahiers de Préhistoire et d'Archéologie III, Genève, Nyon, [s.n.], 1946.

Brunier, I., Mutations de la ville au XVI ${ }^{\mathrm{e}}$, Repli, Extension sur l'eau et fortification de la ville $\mathrm{XVI}^{\mathrm{e}}$, in Broillet, $\mathrm{P}$. (dir.), Les Monuments d'art et d'histoire du canton de Genève, La Genève sur l'eau, Tome 1, Bâle, Wiese, 1997, pp. 56-70.

Carbasse, J.-M., Histoire du droit pénal et de la justice criminelle, Paris, PUF, 2000.

Carbonnier, M., Le droit de punir et le sens de la peine chez Calvin, Revue d'histoire et de philosophie religieuse, tome 54, 1974, pp. 187-201.

Covelle, A., Le Livre des Bourgeois de l'Ancienne République de Genève, Genève, J. Jullien, 1897.

Clasen, C.-P., Anabaptism, a social History, 1525-1618, Ithaca, Londres, Cornell University Press, 1972.

Davidson, N., Sodomy in early modern Venice, in Betteridge, T. (ed.), Sodomy in early modern Europe, Manchester [etc], Manchester Univ. Press, 2002, pp. 67-81.

Dean, T., Crime in medieval Europe 1200-1550, Londres, Harlow [etc.], Longman, 2001. 
Fogel, M., Les cérémonies de l'information dans la France du XVI siècle au milieu du XVIII siècle, Paris, Fayard, 1989.

Foucault, M., Surveiller et punir: naissance de la prison, [1975], Paris, Gallimard, 2005.

Gauvard, C., Jacob, R. (dir.), Les rites de la justice, Gestes et rituels judiciaires au MoyenÂge occidental, Paris, Le Léopard d'Or, 2000.

Geisendorf P.-F. (dir.), Livre des Habitants de Genève 1549-1560, tome 1, Genève, Droz, 1957.

Grosse, C., L'excommunication de Philibert Berthelier. Histoire d'un conflit d'identité, aux premiers temps de la Réforme genevoise (1547-1555), Genève, SHAG, 1995.

Grosse, C., Les Rituels de la Cène, une anthropologie historique du culte eucharistique réformé à Genève (XVIe-XVII siècles), Genève, Droz, 2008.

Grosse, C., La discipline ecclésiastique à Genève, in Backus, I. (dir.) Théodore de Bèze (1519-1605), Actes du Colloque du Genève, septembre 2005, Genève, Droz, 2007.

Hoffmann, C., Social control and the Neighbourhood in European Cities, in Roodenburg, H., Spierenburg, P. (Eds), Social Control in Europe 1500-1800, vol. 1, Ohio state University Press, 2004, pp. 309-327.

Kaden, E.-H., Le jurisconsulte Germain Colladon, ami de Jean Calvin et de Théodore de Bèze, Genève, Georg, 1974.

Lescaze, B., Crimes et Criminels à Genève en 1572, in Collectif, Pour une histoire qualitative: études offertes à Sven Stelling-Michaud, Genève, Presses universitaires romandes, 1975, pp. 45-71.

Lescaze, B., La justice dans le passé, in Lescaze, B., Hirsch, F. (dir.) Encyclopédie de Genève : les institutions politiques, judiciaires et militaires, tome 4, Genève, Association de l'Encyclopédie de Genève, 1991.

Mandrou, R., Magistrats et Sorciers en France au XVIII ${ }^{e}$ siècle, Paris, Seuil, 1980.

Muchembled, R., L'invention de la France Moderne, Monarchie, cultures et sociétés (15001660), Paris, A. Collin, 2002.

Muchembled, R., Le Temps des supplices : de l'obéissance sous les rois absolus, XV $-X V I I I^{e}$ siècles, Paris, Pocket, 2006.

Monter, W., Crime and Punishment in Calvin's Geneva, 1562, in Monter, W., in Enforcing Morality in Early Modern Europe, Londres, Variorum Reprints, 1987a, pp. 281-286.

Monter, W., Sodomy and Heresy in early modern Switzerland, in Monter, W., Enforcing morality in Early Modern Europe, Londres, Variorum Reprints, 1987b, pp. 41-55.

Monter, W., The Consistory of Geneva, 1559-1569, in Monter, W., Enforcing Morality in Early Modern Europe, Londres, Variorum Reprints, 1987c, pp. 467-484.

Mottu-Weber, L., Genève et ses réfugiés, in Migrations vers les villes, Zurich, Chronos, cop. 2000, pp. 157-170.

Naphy, W., Reasonable doubt: defences advanced in early modern sodomy trials in Geneva, in Mulholland, M., Pullan, B., Judicial tribunals in England and Europe, 1200-1700, The trial in history, vol.1, Manchester, New York, Manchester Univ. Press, 2003, pp. 129-145.

Partch, G., Jean Cramer et son précis de l'histoire du droit genevois (1761), Bulletin de la SHAG, tome XIII, 1964, pp 13-87.

Porret, M., Le crime et ses circonstances: de l'esprit de l'arbitraire au siècle des Lumières selon les réquisitoires des procureurs généraux de Genève, Genève, Droz, 1995.

Porret, M., Le crime des filles séduites et abandonnées, in Pestalozzi, J. H., Sur la législation et l'infanticide, Berne [etc.], P. Lang, cop. 2003, pp. 163-187.

Puff, H., Nature on Trial: Acts «Against Nature» in the law courts of Early modern Germany and Switzerland, in Daston, L., Vidal, F. (Eds), The Moral Authority of Nature, Chicago [etc.], The University of Chicago Press, 2004, pp. 232-253. 